\title{
Bispecific Antibody Molecule Inhibits Tumor Cell Proliferation More Efficiently Than the Two-Molecule Combination
}

\author{
Anna-Luisa Volk ${ }^{1}$ (1) Aman Mebrahtu ${ }^{1} \cdot$ Bong-Kook Ko $^{2} \cdot$ Magnus Lundqvist $^{1} \cdot$ Maximilian Karlander $^{1} \cdot$ \\ Hyun-Jong Lee ${ }^{2} \cdot$ Fredrik Y. Frejd $^{3,4} \cdot \mathrm{Kyu}^{-T a e} \mathrm{Kim}^{2} \cdot$ Jong-Seo Lee $^{2} \cdot$ Johan Rockberg $^{1}$
}

Accepted: 10 February 2021 / Published online: 15 March 2021

(c) The Author(s) 2021

\begin{abstract}
Background Monoclonal antibodies (mAbs) have proved to be a valuable tool for the treatment of different cancer types. However, clinical use of an increasing number of $\mathrm{mAbs}$, have also highlighted limitations with monotherapy for cancers, in particular for such with more complex mechanisms, requiring action on additional molecules or pathways, or for cancers quickly acquiring resistance following monotherapy. An example for the latter is the mAb trastuzumab, FDA approved for treatment of metastatic gastric carcinoma. To circumvent this, researchers have reported synergistic, anti-proliferative effects by combination targeting of HER2 and EGFR by trastuzumab and the EGFR-targeting mAb Cetuximab overcoming trastuzumab resistance.

Methods Maintaining the proven functionality of trastuzumab, we have designed bi-specific antibody molecules, called AffiMabs, by fusing an EGFR-targeting Affibody molecule to trastuzumab's heavy or light chains. Having confirmed binding to EGFR and Her2 and cytotoxicity of our AffiMabs, we analyzed apoptosis rate, receptor surface levels, phosphorylation levels of receptors and associated signaling pathways as well as differentially expressed genes on transcriptome level with the aim to elucidate the mode of action of our AffiMabs.

Results The AffiMabs are able to simultaneously bind HER2 and EGFR and show increased cytotoxic effect compared to the original trastuzumab therapeutic molecule and, more importantly, even to the combination of trastuzumab and EGFRtargeting Affibody molecule. Analyzing the mode of action, we could show that bi-specific AffiMabs lead to reduced surface receptor levels and a downregulation of cell cycle associated genes on transcriptome level.

Conclusion Our study shows that transcriptome analysis can be used to validate the choice of receptor targets and guide the design of novel multi-specific molecules. The inherent modularity of the AffiMab format renders it readily applicable to other receptor targets.
\end{abstract}

\section{Introduction}

Monoclonal antibodies (mAbs) are approved oncology therapeutics with clinical efficacy in different tumor types. As a common strategy in cancer therapy, many mAbs are designed to target surface receptors on the cancer cell. A popular and well-studied class of receptors is the ErbB

Johan Rockberg

johan.rockberg@biotech.kth.se

1 School of Engineering Sciences in Biotechnology, Chemistry and Health, AlbaNova University Center, KTHRoyal Institute of Technology, 106 91, Stockholm, Sweden

2 AbClon Inc., Guro-dong, Guro-gu, Seoul 152-779, Korea

3 Affibody AB, 17169 Solna, Sweden

4 Department of Immunology, Genetics and Pathology, Uppsala University, 75105 Uppsala, Sweden family of receptor tyrosine kinases. Aberrant expression and signaling of ErbB receptors have long been associated with tumorigenesis of various types of solid tumors [1], making these receptors attractive therapeutic targets. ErbB receptors play an important role in the proliferation and differentiation of epithelial, mesenchymal, and neuronal cells $[1,2]$. The four family members EGFR/ERBB1/HER1, ERBB2/ HER2/NEU, ERBB3/HER3, and ERBB4/HER4 have similar structures, consisting of an extracellular domain (ECD), a single-pass transmembrane domain, and an intracellular kinase domain. Ligand binding stimulates homo- or heterodimerization, which leads to receptor activation and signaling through various downstream signaling cascades with the extracellular signal-regulated kinase (ERK)-mitogen-activated protein kinase (MAPK) and protein kinase $\mathrm{B}$ (AKT)-phosphoinositide 3 kinase (PI3K) being two of the most well-known pathways regulating cell proliferation, 


\section{Key Points}

We have developed a new bispecific antibody format called AffiMabs, consisting of an Affibody molecule fused to an antibody's heavy or light chain, a modular format that allows for easy adaptation to different targets.

The model AffiMab in this study, an epidermal growth factor receptor (EGFR)-binding Affibody fused to the therapeutic human epidermal growth factor receptor 2 (HER-2)-binding antibody trastuzumab, had a stronger cytotoxic effect on a gastric cancer cell line than the combination of both parental molecules.

The mode of action of this AffiMab is suggested to be a combination of reduced surface receptor levels and a downregulation of transcription of cell cycle-associated genes.

differentiation, apoptosis, and migration. Epidermal growth factor receptor (EGFR) and human epidermal growth factor receptor 2 (HER2) are the most well-studied in this receptor family [1], and this has resulted in several mAbs being approved for cancer therapy to date, including cetuximab, panitumumab, and necitumumab (binding EGFR) and trastuzumab and pertuzumab (targeting HER2).

Trastuzumab (Herceptin) is a humanized mAb approved for the treatment of HER2-positive metastatic breast cancer and gastric cancer. Trastuzumab treatment is effective in patients with HER2-positive disease, especially in combination with chemotherapy, and has proven superior to chemotherapy alone $[3,4]$, but the therapeutic effect is often transient, implying the occurrence of trastuzumab resistance [5]. Compensatory signaling through other receptors of the ErbB family and through autocrine ligand expression have been identified as mechanisms of trastuzumab resistance [5-7]. Several studies have suggested that upregulation of EGFR expression is a factor that contributes to acquired trastuzumab resistance [8-10], which paved the way for therapeutic strategies targeting both receptors simultaneously. Increased antitumor effects were reported for various tumor types and even for trastuzumab-resistant cells when combining the EGFR-binding mAb cetuximab with trastuzumab [8, 11-13] or trastuzumab with other HER2-targeting agents [14, 15].

However, a clear drawback with combination therapy is the elevated costs for parallel development, production, and approval [16]. Bispecific antibodies have emerged as a strategy to circumvent this by binding different targets with only one molecule. The aforementioned knowledge about HER pathology encouraged us to develop a bispecific EGFR/ HER2 antibody. To this end, we fused an EGFR-binding
Affibody molecule to the C-terminus of trastuzumab's heavy chain (HC) or light chain (LC) to generate a socalled AffiMab. Affibody molecules-6 kDa alpha-helical engineered scaffolds (for a detailed review see Ståhl et al. [17]) - have been employed previously to generate bispecific binders. Insulin-like growth factor (IGF)-1R-, HER3-, and interleukin-6-binding Affibody molecules have been fused to trastuzumab, cetuximab, and adalimumab (Humira), respectively, with promising in vitro results [18, 19]. Fusion proteins of EGFR- and HER2-binding Affibody molecules have also been investigated and found to have no effect on cell proliferation [20, 21]. Compared with these bispecific Affibody fusions, the AffiMab format presented here has the advantage of bivalent expression of both binding entities while retaining the functionalities of the scaffold antibody.

Here, we report on the generation of a HER2-EGFRbinding AffiMab and the functional characterization of the $\mathrm{HC}$ and LC fusions. We conducted binding and cell proliferation studies. The increased cytotoxic effect of the AffiMabs encouraged us to further elucidate the mechanism of action of these molecules. We analyzed apoptosis rate, receptor surface levels, phosphorylation levels of receptors, and associated signaling pathways, as well as differentially expressed genes at the transcriptome level to explain the observed effect.

\section{Methods}

\subsection{Cell Lines and Antibodies}

The gastric carcinoma cell line NCI-N87 was purchased from ATCC (CRL-5822 ${ }^{\mathrm{TM}}$ ) and maintained in RPMI-1640 medium (Sigma) supplemented with $10 \%$ fetal bovine serum (FBS) (Sigma). A-431 cells (ATCC ${ }^{\circledR}$ CRL-1555) were cultured in Dulbecco's Modified Eagle's Medium supplemented with $10 \%$ FBS. SKOV3 cells were cultured in McCoy's 5a medium, supplemented with $10 \%$ FBS. All cells were maintained in a humidified atmosphere with $5 \%$ carbon dioxide at $37{ }^{\circ} \mathrm{C}$ and passaged every $2-3$ days.

Trastuzumab (Roche, Basel, Switzerland) and the immunoglobulin G (IgG) control adalimumab (AbbVie, North Chicago, IL, USA) were purchased from a local pharmacy. The EGFR-binding Affibody molecule $\mathrm{Z}_{1907}$, henceforth referred to as zEGFR, was obtained from Affibody AB. Both AffiMab variants were secreted from HEK-293F cells as described in the following subsections.

\subsection{Cloning and Production of AffiMabs}

AffiMabs were constructed by fusion of the gene for the EGFR-binding Affibody molecule zEGFR to the C-terminus of the HC or LC of trastuzumab via a 15-amino-acid 
glycine-serine linker $\left(\mathrm{SS}(\mathrm{G})_{4} \mathrm{~S}(\mathrm{G})_{6} \mathrm{SS}\right.$; Fig. $\mathrm{S} 1$ in the electronic supplementary material [ESM]). The HC and LC constructs were cloned into pcDNA3.3 and pOptiVEC vectors, respectively, using solid-phase cloning as previously described [22]. In brief, the insert sequences for antibody, HC, LC, and Affibody with linker were polymerase chain reaction amplified in separate reactions and sequentially immobilized and hybridized on streptavidin-coated magnetic beads. AffiMabs were transiently produced using the $293 \mathrm{~F}$ system (Invitrogen) and purified using protein-A chromatography (GE Healthcare, Uppsala, Sweden).

Two AffiMabs, Tra-zEGFR-L15 (see Sect. 3.1), and the isotype control Ada-zEGFR-L15 were at a later stage produced employing the transient ExpiCHO expression system. To this end, the HC and LC of the AffiMabs were cloned by conventional restriction cloning into a dual-expression cassette vector system pKTH17 (Fig. S2 in the ESM), encoding both the $\mathrm{HC}$ and the LC. All constructs were cultured and expressed in ExpiCHO cells (Thermo Fisher Scientific, Waltham, MA, USA) according to the ExpiCHO standard titer protocol (ExpiCHO Expression System User Guide, Pub. No. MAN0014337 Rev. C.0). Protein A-facilitated purification was performed on an ÄktaSTART system on mAb SelectSuRe columns (GE Healthcare).

\subsection{Measurement of Target Binding by Surface Plasmon Resonance}

To measure target binding, HER2-ECD-His was immobilized onto a CM5 sensor chip surface (GE Healthcare) using amine coupling at approximately 1000 response units. Antibody $(3 \mu \mathrm{g} / \mathrm{mL})$ and EGFR-ECD-His $(40 \mu \mathrm{g} / \mathrm{mL})$ protein were sequentially injected by binding for $2 \mathrm{~min}$ and stabilization for $3 \mathrm{~min}$ at $50 \mu \mathrm{L} / \mathrm{min}$ flow rate. For affinity measurement, a slightly modified set-up was used; goat anti-human $\operatorname{IgG}(\gamma)$ (Invitrogen) was immobilized onto a CM5 sensor chip using amine coupling, and antibodies were captured. Then, HER2-ECD-His or EGFR-ECD-His protein was injected at concentrations ranging from 0 to $320 \mathrm{nM}$ or from 0 to $500 \mathrm{nM}$, respectively. Sensorgrams were obtained at each concentration and evaluated using BIAevaluation software.

\subsection{Measurement of Target Binding on Cells}

A-431 and SKOV3 cells were harvested by trypsinization, neutralized with complete growth medium, and washed once with phosphate buffered saline (PBS); $1.5 \times 10^{5}$ cells were labeled with trastuzumab, AffiMabs, or zEGFR Affibody molecule, respectively, at a concentration of 1 or $0.01 \mu \mathrm{g} / \mathrm{mL}$ Affibody molecule while shaking for $45 \mathrm{~min}$ at room temperature. Cells were washed and then labeled with $2 \mu \mathrm{g} / \mathrm{mL}$ goat anti-human IgG-Alexa488 or goat anti-Affibody-Alexa488 for 30 min on ice in the dark. After one final wash with PBS supplemented with $1 \%$ bovine serum albumin (PBS-B), cells were analyzed on a Gallios flow cytometer (Beckman Coulter).

\subsection{Cell Proliferation Assay}

NCI-N87 cells were seeded on a tissue culture-treated 96-well plate (Nunc) at a density of 100,000 cells $/ \mathrm{mL}$ in 100 $\mu \mathrm{L}$ growth medium. After $24 \mathrm{~h}$, the medium was exchanged for medium containing AffiMabs or controls at eight different concentrations varying from 133 to $0.008 \mathrm{nM}$. Controls were trastuzumab, a nonbinding IgG, adalimumab (Humira), an isotype control consisting of adalimumab with zEGFR coupled to the LC (Ada-zEGFR-L15), mono- and bivalent zEGFR, and a mixture of mono- or bivalent zEGFR with trastuzumab. For the monovalent zEGFR Affibody molecule, double amounts, i.e., $266-0.016 \mathrm{nM}$, were used to compensate for the bivalency of the AffiMabs. However, for simplified visualization and better comparability in the diagram, zEGFR was plotted at the corresponding dilution step of the antibodies. Cells were cultured for 4 days at $37^{\circ} \mathrm{C}$ in a humidified atmosphere with $5 \% \mathrm{CO}_{2}$ in a plastic bag to minimize evaporation. On day 4 , we added $10 \mu \mathrm{L}$ alamarBlue ${ }^{\circledR}$ (Thermo Fisher Scientific) to each well. After $4 \mathrm{~h}$ incubation in the conditions described, fluorescence at an excitation wavelength of $530 \mathrm{~nm}$ and emission wavelength of $590 \mathrm{~nm}$ was measured.

\subsection{Antibody Treatment of NCI-N87 Cells for Further Analysis}

NCI-N87 cells were seeded in a tissue culture-treated multiwell plate (VWR International) at a density of 120,000 cells/ $\mathrm{mL}$ growth medium. After $24 \mathrm{~h}$, the medium was exchanged for medium containing $133 \mathrm{nM}$ of antibody, Affibody molecule, or AffiMab, respectively. Cells were cultured for 2-4 days at $37{ }^{\circ} \mathrm{C}$ in a humidified atmosphere with $5 \% \mathrm{CO}_{2}$ in a plastic bag to minimize evaporation.

\subsection{Receptor Surface-Level Analysis by Flow Cytometry}

NCI-N87 cells were cultured in a $24-w e l l$ plate (VWR International) in $500 \mu \mathrm{L}$ growth medium as described in the previous paragraph. On day 4 , cells were harvested by trypsination and washed once with PBS. Per sample, onehalf of the cells were incubated with the mouse anti-EGFR antibody ab30 (Abcam) at a concentration of $1 \mu \mathrm{g} / \mathrm{mL}$ on ice. Meanwhile, the other half was incubated with PBS-B. After washing the cells once with PBS-B, the anti-EGFRlabeled cells were incubated with secondary antibodies goat anti-mouse IgG Alexa488 (Molecular Probes) and goat 
anti-human IgG-Alexa647 (Molecular Probes) at $2 \mu \mathrm{g} / \mathrm{mL}$ for $1 \mathrm{~h}$ on ice in the dark. The cells previously only treated with PBS-B were incubated with an anti-HER2 Affibody dimer (Z00477) ${ }_{2}$-Cys (Affibody AB) fused to fluorescein isothiocyanate at $0.38 \mu \mathrm{g} / \mathrm{mL}$ and a goat anti-human IgGAlexa647 (Molecular probes) at $2 \mu \mathrm{g} / \mathrm{mL}$, likewise for $1 \mathrm{~h}$ on ice in the dark. After one wash with PBS-B, surface receptor levels were analyzed using flow cytometry.

In a similar fashion, untreated NCI-N87 cells were first incubated with AffiMabs or controls while kept on ice and then stained for fluorescence-activated cell sorting (FACS) analysis as described to evaluate the effect of competition between the different binders. A detailed protocol can be found in the ESM.

\subsection{Messenger RNA Isolation and Transcriptomic Analysis}

For messenger RNA (mRNA) isolation, NCI-N87 cells were cultured on a tissue culture-treated 6-well plate (Greiner BioOne BioScience) in $2.5 \mathrm{~mL}$ growth medium as described in Sect. 2.6. After $48 \mathrm{~h}$, cells were harvested using trypsinization and washed once with PBS, and total RNA was isolated using the Qiagen RNeasy Plus Mini kit according to the manufacturer's instructions. If RNA isolation was not performed on the day of cell harvest, the washed cell pellet was resuspended in $250 \mu \mathrm{L}$ RNAlater ${ }^{\circledR}$ Stabilization Solution (Ambion) and stored at $-80^{\circ} \mathrm{C}$. RNA sequencing was performed at GATC Biotech AG.

Kallisto [23] with a complementary DNA (cDNA) human reference (GRCh38, Ensemble release 92) as index file was used to quantify transcript abundances. The heatmap with sample-to-sample distances was based on Euclidean distances of variance stabilizing transformations of transcript counts. The R package DESeq 2 [24] with transcript counts was used for differential expression analysis. PIANO [25] was used to perform gene set enrichment analysis (GSEA), with the adjusted $p$-values and $\log _{2}$-fold changes from the differential expression analysis and the KEGG gene sets (C6, version 6.1) downloaded from MSigDB [26]. Pathways in Fig. S3 (see the ESM) were created with Pathview [27], and their colors were subsequently adjusted from red/green to red/blue to ensure accessibility for people who are color blind.

\section{Results}

\subsection{Solid-Phase Cloning Generates Full-Length AffiMabs}

zEGFR was genetically fused via a 15 -amino acid glycine-serine linker to either the $\mathrm{LC}$ or the $\mathrm{HC}$ of the $\mathrm{mAb}$ trastuzumab employing our solid-phase cloning technique [22]. Solid-phase cloning does not depend on the use of restriction enzymes for the fusion of genetic elements but instead is based on the hybridization of vector elements on magnetic streptavidin-coated beads, which allows a very modular assembly of the different vector parts (Fig. S1 in the ESM). The zEGFR molecule was fused to the C-terminus of either antibody chain, generating bispecific and bivalent AffiMabs towards HER2 and EGFR, termed Tra-zEGFRL15 (for the LC fusion) and Tra-zEGFR-H15 (for the HC fusion) (Fig. 1a). The AffiMabs were purified from HEK$293 \mathrm{~F}$ supernatants using protein-A chromatography and analyzed using sodium dodecyl sulphate-polyacrylamide gel electrophoresis (Fig. 1b). A clear size shift corresponding to the size of the Affibody molecule plus linker was detected on the LC of Tra-zEGFR-L15 and on the HC of Tra-zEGFR$\mathrm{H} 15$, indicating successful fusion. The other antibody chain corresponded in size to that of trastuzumab, indicating successful production of full-length AffiMabs.

\subsection{AffiMabs Recognize Soluble and Natural Targets on Cells as well as Fragment Crystallizable Receptor}

Having successfully produced AffiMabs, we next sought to demonstrate their functionality. The binding affinity of the AffiMabs to their soluble targets, the ECDs of HER2 and EGFR, was analyzed using surface plasmon resonance (SPR) and compared with that of trastuzumab. To this end, HER2-ECD was immobilized on an SPR sensor chip and sequentially exposed to the AffiMabs or trastuzumab and EGFR-ECD. Both AffiMabs bound HER2-ECD with affinity equal to that of trastuzumab (Fig. 1c; Table S1 in the ESM). The addition of EGFR-ECD led to another mass gain for both AffiMabs, indicative of simultaneous binding to EGFR and HER2. However, Tra-zEGFR-L15 showed a slightly higher affinity towards EGFR than did Tra-zEGFR-H15 (Fig. 1c; Table S1 in the ESM). Interestingly, compared with free zEGFR, which was reported to have a $K_{D}$ of $5.4 \mathrm{nM}$ [28], the affinity of the trastuzumab-fused zEGFR decreased by approximately one order of magnitude (Table S1 in the ESM). As anticipated, trastuzumab did not bind EGFR.

In the next step, we analyzed the ability of the AffiMabs to recognize their natural targets on different cancer cell lines. For this purpose, the epidermoid carcinoma cell line A-431 and the ovarian carcinoma cell line SKOV3 were incubated with either AffiMab or trastuzumab or zEGFR alone. A-431 cells express high amounts of EGFR and moderate amounts of HER2, whereas SKOV3 cells express high levels of HER2 and lower levels of EGFR [29]. Binding was detected using flow cytometry after staining with a secondary fluorescently labeled anti-human IgG or anti-Affibody antibody, respectively. Both AffiMabs showed elevated binding to A-431 
a

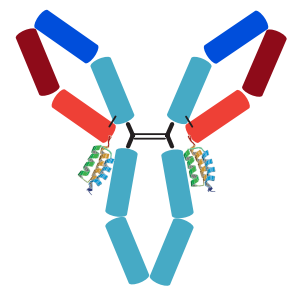

TRA-ZEGFR-L15

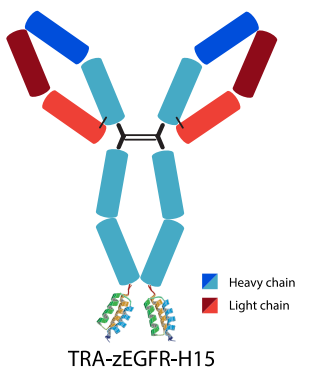

TRA-ZEGFR-H15 b

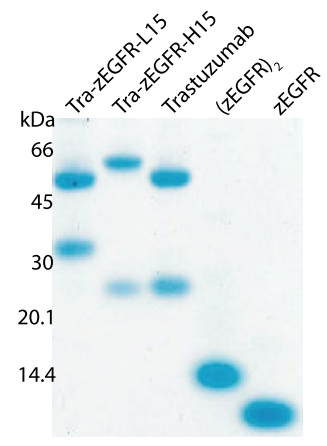

C

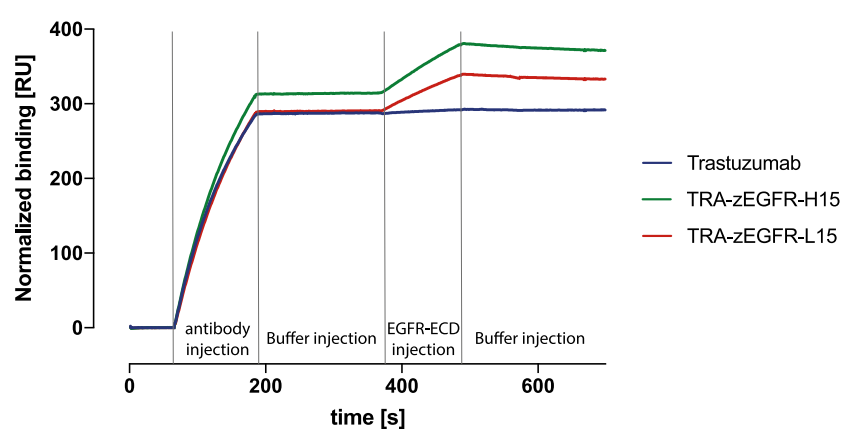

d

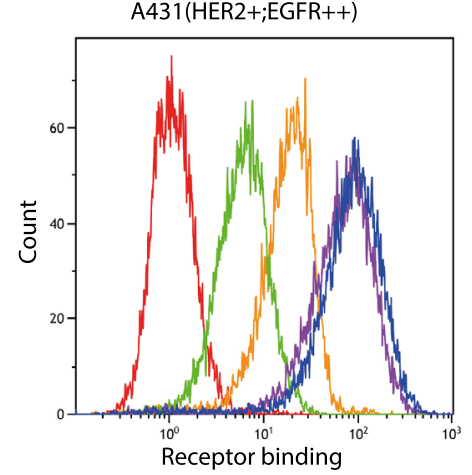

Fig. 1 Generation of fully functional, bispecific AffiMabs. a Schematic illustration of the two AffiMabs analyzed in this study; trastuzumab with the EGFR-binding Affibody molecule fused to the light chain (left), Tra-zEGFR-L15, and trastuzumab with a heavy chain fusion (right), Tra-zEGFR-H15. b Comparison of the AffiMabs with trastuzumab on reducing sodium dodecyl sulphate-polyacrylamide gel electrophoresis shows a shift for the heavy or light chain, respectively, corresponding to the size of the zEGFR Affibody molecule, including linker, which demonstrates the successful fusion of the Affibody molecule to trastuzumab. c HER2-ECD was immobilized on a surface plasmon resonance chip and subjected to subsequent flows of AffiMabs or trastuzumab, respectively, and EGFR-ECDs. All three molecules showed comparable binding to HER2. As expected, trastuzumab was unable to bind EGFR. The heavy chain fusion TrazEGFR-H15 shows slightly weaker binding to EGFR than the light

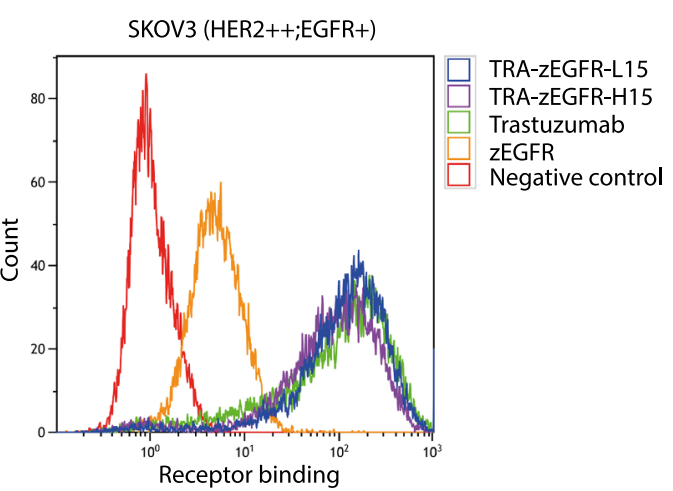

chain fusion Tra-zEGFR-L15. d Binding to the natural target was tested by incubating the AffiMabs and trastuzumab with the cancer cell lines A-431 and SKOV3 and measuring the amount of bound antibody by flow cytometry. The AffiMabs (dark blue and violet) show strong binding to the EGFR overexpressing cell line A-431 (left). The peak shift to the right indicates stronger cell binding for the AffiMabs than the Affibody molecule (orange) or trastuzumab (green) alone because of their ability to bind both EGFR and HER2. For HER2-overexpressing SKOV3 cells, binding of the AffiMabs was comparable to that of trastuzumab. The Affibody molecule zEGFR alone showed low levels of cell binding. ECD extracellular domain, $E G F R$ epidermal growth factor receptor, HER2 human epidermal growth factor receptor $2, R U$ response units, $z E G F R$ EGFR-binding Affibody molecule $\mathrm{Z}_{1907}$ 
cells compared with trastuzumab or zEGFR, indicating a favorable effect of bispecificity on cell binding (Fig. 1d). However, for the HER2-overexpressing SKOV3 cell line, AffiMabs exhibited equally strong binding as trastuzumab but much stronger binding than zEGFR. Given the low levels of EGFR compared with HER2 on SKOV3 cells, the influence of EGFR binding on AffiMab binding is likely to be marginal. Yet, these results show that the Affibody fusions do not affect trastuzumab's ability to bind HER2.

Since the fragment crystallizable $(\mathrm{Fc})$-region of an antibody fulfills important functions for antibody recycling, it was important to ensure that the C-terminal HC-fusion did not affect this function. To this end, we compared the ability of both Tra-zEGFR-H15 and trastuzumab to bind the neonatal $\mathrm{Fc}$ receptor $(\mathrm{FcRn})$, which is involved in antibody recycling. Hela cells were stably transfected to express FcRn-enhanced green fluorescent protein fusions. Cells were incubated with trastuzumab- or Tra-zEGFR-H15-containing cell supernatants at $\mathrm{pH} 6$ and $\mathrm{pH} 8$ and stained with fluorescently labeled goat anti-human IgG. Binding of Tra-zEGFRH15 to FcRn at pH 6 was comparable to that of trastuzumab (Fig. S4 in the ESM). The minor difference in binding is likely to be due to variations in antibody concentration because cell supernatants were used. No binding of the AffiMab could be detected at $\mathrm{pH} 8$ (data not shown), indicating that the effect of HER2 or EGFR binding is negligible.

In summary, both AffiMabs recognized their targets, both as soluble molecules and on the surface of cells, and could bind them simultaneously. The Affibody fusions do not affect HER2- or FcRn binding.

\subsection{AffiMabs Elicit an Increased Cytotoxic Effect}

After having established the ability of AffiMabs to recognize both targets, we next analyzed the effect of AffiMabs on tumor cell growth. For this purpose, gastric carcinoma NCIN87 cells were incubated with increasing concentrations of AffiMab, trastuzumab, and controls. After a 4-day incubation, cell proliferation was assessed using the alamarBlue assay for monitoring of viable cells. Bivalent zEGFR, the tumor necrosis factor (TNF)-binding mAb adalimumab (IgG control), and an adalimumab-based AffiMab, Ada-zEGFRL15 (isotype control), did not affect cell growth, but a reduction in cell proliferation could be seen for trastuzumab, the combination of trastuzumab and bivalent zEGFR, and both AffiMabs at concentrations above 2 nM (Fig. 2). Strikingly, the AffiMab molecule reduced cell growth further than trastuzumab or the combination of trastuzumab and bivalent zEGFR. Cell proliferation was decreased to $35 \%$ of an uninhibited control after treatment with $133 \mathrm{nM}$ of Tra-zEGFR-L15, whereas the addition of the same amount of trastuzumab or trastuzumab with bivalent zEGFR only reduced cell proliferation to 56 and $53 \%$, respectively. This

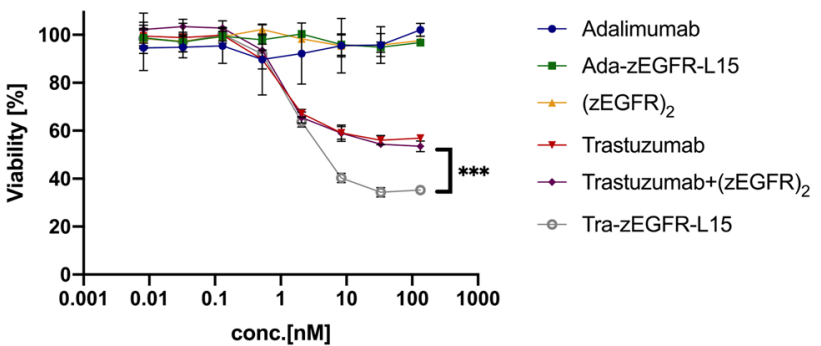

Fig. 2 AffiMabs show greater cytotoxic effect than trastuzumab. NCIN87 cells were incubated with different concentrations of AffiMab (gray), trastuzumab (red), bivalent zEGFR (yellow) or a combination thereof (violet). The tumor necrosis factor-binding monoclonal antibody adalimumab (blue) and an adalimumab-based AffiMab, AdazEGFR-L15 (green), were used as immunoglobulin G and isotype control, respectively. After 4 days, proliferation was measured with the addition of alamarBlue. The controls and bivalent zEGFR did not affect the number of viable cells. Addition of trastuzumab with or without (zEGFR) 2 reduced cell proliferation to around 53\%. Both AffiMabs decreased cell growth even further, indicating a synergistic effect of simultaneous HER2 and EGFR binding. The average and standard deviation of triplicates are shown. Cell growth after addition of phosphate buffered saline was set to $100 \%$. conc. concentration, $E G F R$ epidermal growth factor receptor, HER2 human epidermal growth factor receptor 2, $z E G F R$ EGFR-binding Affibody molecule $\mathrm{Z}_{1907}$

indicates that the fusion of zEGFR to trastuzumab conferred cytotoxic properties to the AffiMab molecules that could not be achieved by simply mixing the two components. Interestingly, no differences in cytotoxic potential were observed between the two AffiMabs (Fig. S5 in the ESM), suggesting that the fusion site does not affect cytotoxicity.

\subsection{AffiMabs Reduce Surface Levels of Human Epidermal Growth Factor Receptor 2 (HER2) and Epidermal Growth Factor Receptor}

Having observed an increased cytotoxic effect of both AffiMabs compared with a combination treatment of trastuzumab and zEGFR, we aimed to further elucidate the mechanism of action of these bispecific molecules. First, we looked at the cell surface levels of HER2 and EGFR on NCI-N87 cells after a 4-day exposure to $133 \mathrm{nM}$ of the different binders, which was the concentration that gave rise to the most distinct differences in the proliferation experiment. To assess HER2 surface levels, treated cells were stained with a fluorescently labeled HER2-binding Affibody dimer on ice, which does not compete with trastuzumab for binding to HER2 (see the Methods and Fig. S6a in the ESM). Binding measurement of the anti-HER2 Affibody dimer by flow cytometer showed a 35\% signal reduction for samples treated with trastuzumab, trastuzumab combined with zEGFR, and both AffiMabs compared with an untreated control, the IgG 


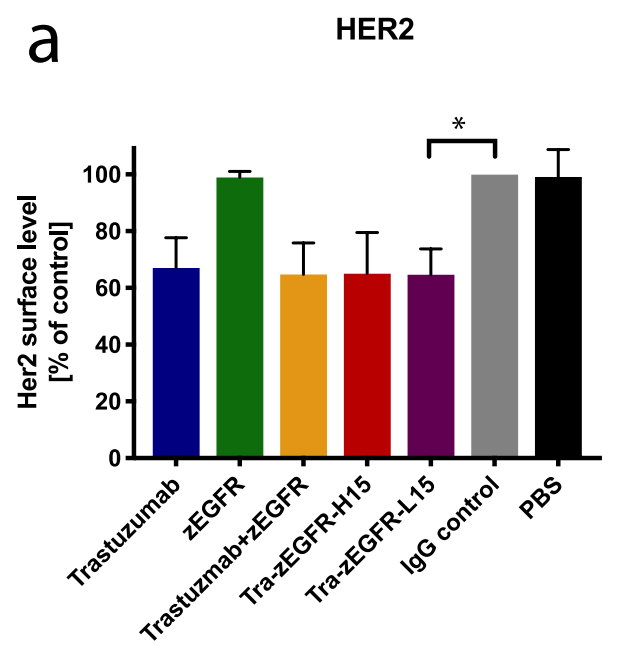

Fig. 3 AffiMabs reduce receptor surface levels. NCI-N87 cells were incubated with $133 \mathrm{nM}$ of the different binders, and, again, adalimumab was used as IgG control. a HER2 surface levels after a 4-day incubation were measured in FACS by a fluorescently labeled, HER2-binding Affibody dimer, which does not compete with trastuzumab for binding. Compared with the IgG control, HER2 surface levels in AffiMab-treated samples were equally reduced (paired $t$ test; $* p<0.05$ ) as samples treated with trastuzumab or a combination of trastuzumab and zEGFR (paired $t$ test; non-significant). The means and standard deviations of three independent experiments are shown. HER2 surface levels in IgG control samples were set to $100 \%$. b EGFR surface levels after a 4-day incubation were measured in FACS by a second EGFR-binding monoclonal antibody. A certain

control or zEGFR-treated cells (Fig. 3a). Both AffiMabs reduced HER2 surface levels equally as well as trastuzumab.

A similar experiment was performed to look at EGFR surface levels. For this, the treated cells were stained with a mouse anti-EGFR mAb, which was detected with a fluorescently labeled anti-mouse IgG. A reduction in binding signal could be observed for samples treated with zEGFR, trastuzumab combined with zEGFR, and both AffiMabs compared with an untreated control, an IgG control, or trastuzumab-treated cells (Fig. 3b). However, signal reduction also occurred after the competition analysis, where NCIN87 cells were sequentially incubated with the AffiMabs or controls, followed by the anti-EGFR mAb and the secondary antibody for only $1 \mathrm{~h}$ on ice to prevent receptor internalization (see the Methods and Fig. S6b in the ESM). This suggested that the anti-EGFR mAb and zEGFR have at least partly overlapping epitopes or sterically hinder each other. Attempts to remove the AffiMabs and the control binders from the cell surface using acid washes prior to labeling with the anti-EGFR mAb remained unsuccessful. However, a direct comparison of the signal reduction brought about by epitope competition (Fig. S6b in the ESM) and the signal reduction observed after a 4-day incubation (Fig. 3b) revealed a significant difference. While competitive binding accounts for the reduction in signal observed for zEGFR

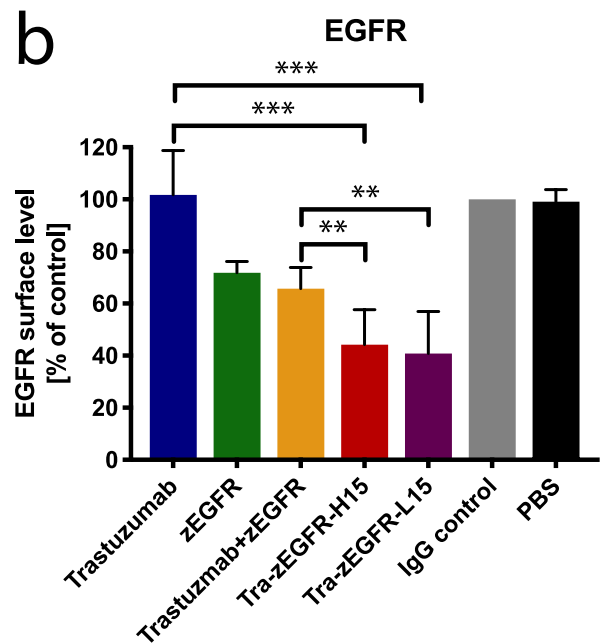

degree of binding competition with zEGFR was observed, which accounts for the reduced levels measured for zEGFR and zEGFR with trastuzumab. The further reduction in EGFR surface levels elicited by the AffiMabs is significant and could be ascribed to the simultaneous engagement of EGFR and HER2 (paired $t$ test; $* * * p<0.0001$; $* * p<0.01$ ). No effect on EGFR levels could be detected after treatment with trastuzumab. The means and standard deviations of six independent experiments are shown. HER2 surface levels in IgG control samples were set to $100 \%$. EGFR epidermal growth factor receptor, FACS fluorescence-activated cell sorting, HER2 human epidermal growth factor receptor $2, I g G$ immunoglobulin $\mathrm{G}, P B S$ phosphate buffered saline, $z E G F R$ EGFR-binding Affibody molecule $\mathrm{Z}_{1907}$

and trastuzumab in combination with zEGFR, the further reduction in binding signal measured for both AffiMabs cannot be explained by competition for binding to EGFR. This reduction by more than 20 percentage points compared with trastuzumab with EGFR is significant $(t$ test, $p<0.005$ ) and thus suggests a biological cause. Previously, receptor clustering and subsequent increased internalization with impaired recycling has been mentioned as an explanation for reduced receptor levels brought about by multispecific antibodies [30]. Unfortunately, confocal microscopy analysis did not show any distinct differences in receptor expression or localization on the cells imaged for our AffiMabs compared with an IgG control (Fig. S7 in the ESM).

\subsection{Cytotoxic Effect of AffiMabs is Associated with Downregulation of Housekeeping Genes at the Transcript Level}

To understand what rendered the AffiMab molecules superior to trastuzumab in the cytotoxicity study, we performed a transcriptome analysis of the cells. RNA was isolated from NCI-N87 cells after a 2-day treatment with AffiMab molecules, trastuzumab, zEGFR, trastuzumab with zEGFR, Humira, or PBS, and a cDNA library of all mRNAs was sequenced. Cells treated with trastuzumab or any of the 
trastuzumab-based AffiMab molecules showed an overall high transcriptome similarity (Fig. S8 in the ESM).

This similarity was also demonstrated by differential expression analysis between untreated cells, i.e., only PBS was added, and samples treated with Tra-zEGFR-H15, TrazEGFR-L15, or trastuzumab. In this analysis, the majority of significantly (adjusted $p<0.01$ ) up- and downregulated genes were shared between AffiMab- and trastuzumabtreated cells compared with nontreated cells (Fig. S9a,b in the ESM); however, the AffiMabs appeared to induce a more intense effect as a larger portion of these genes displayed a doubling or halving of gene expression (Fig. S9c,d in the ESM).

A GSEA was performed to analyze differences in pathway regulation between the different treatments rather than specific genes.

As we were interested in understanding the increased cytotoxicity observed for our AffiMabs compared with trastuzumab, the GSEA focused on these three binders. The decrease in cell proliferation seen for the AffiMabs in the alamarBlue assay (Fig. 2) was also reflected in the GSEA. Here, many pathways related to housekeeping functions and proliferation, such as DNA replication and repair, glycolysis, and cell cycle, were downregulated in cells treated with Tra-zEGFR-H15 or Tra-zEGFR-L15 compared with cells treated with trastuzumab (Fig. 4; Fig. S10 in the ESM). Interestingly, pathways related to apoptosis were not significantly changed. This observation could be confirmed by a flow cytometric analysis of cells that had undergone different treatments. Treated cells were stained with annexin V and propidium iodide to differentiate apoptotic from necrotic cells (Methods section in the ESM). Flow cytometric analysis revealed a slight but nonsignificant increase in percentage of apoptotic cells for samples treated with AffiMabs compared with treatment with trastuzumab, monovalent zEGFR, or adalimumab (Fig. S11 in the ESM), suggesting that induction of apoptosis is not the main driving force for the observed cytotoxic effect of the AffiMabs.

In summary, the transcriptome analysis revealed that, upon treatment with AffiMabs, pathways associated with cell metabolism, cell cycle, and protein synthesis were downregulated, which could explain the increased cytotoxic effect seen with AffiMab treatment.

\subsection{Transcriptome Analysis Indicates Cellular Adaptation via HER3 Transcript Upregulation}

The GSEA also indicated that some pathways were also upregulated in the AffiMab-treated samples compared with trastuzumab (Fig. S10 in the ESM). Several of those pathways have been associated with cancer, e.g., the notch signaling pathway. Interestingly, the ErbB pathway was recognized as significantly upregulated after treatment with Tra-zEGFR-H15 compared with trastuzumab. While this pathway is not listed among the significantly upregulated pathways after treatment with Tra-zEGFR-L15, when looking at gene level, most of the significantly upregulated ErbB pathway genes for Tra-zEGFR-H15 were also upregulated for Tra-zEGFR-L15 (Table S2, Fig. S3 in the ESM). Compared with trastuzumab, HER3/ErbB3 and PI3K in particular

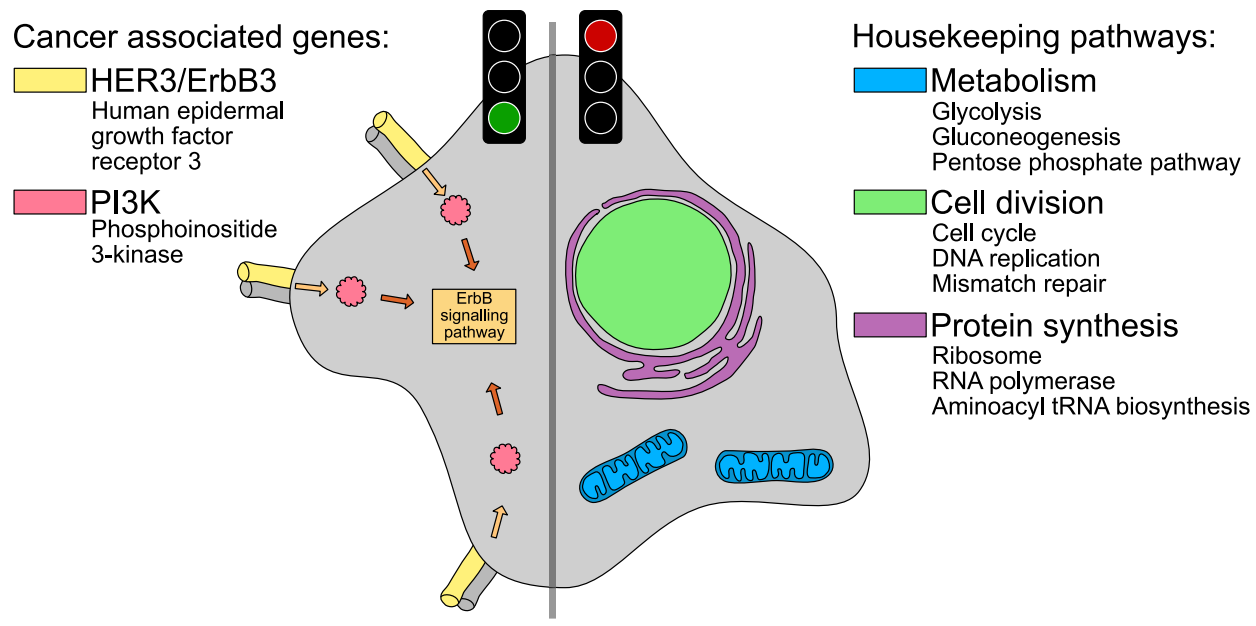

Fig. 4 AffiMab treatment induces downregulation of housekeeping pathways and upregulation of cancer-related pathways. Differential expression and a gene set enrichment analysis was carried out on cells treated with AffiMabs, trastuzumab, and controls. The ErbB pathway was significantly upregulated after treatment with any of the two AffiMabs compared with trastuzumab. On the gene level, notably HER3/ErbB3 and PI3K were significantly upregulated, whereas EGFR/ErbB1, HER2/ErbB2, and HER4/ErbB4 were not. Housekeep- ing function pathways were significantly downregulated. Among these were metabolic pathways such as the glycolysis and pentose phosphate pathways, cell division pathways such as cell cycle and DNA replication, and protein synthesis pathways, such as ribosome and RNA polymerase. EGFR epidermal growth factor receptor, $H E R 3$ human epidermal growth factor receptor $3, P 13 \mathrm{~K}$ phosphoinositide 3 kinase 
were upregulated after treatment with either AffiMab. However, we could not detect any significant change in transcript level for AKT and ERK/MAPK1. Increased expression of HER3 and signaling through the PI3K/AKT pathway is known as an escape pathway upon EGFR or HER2 inhibition [31, 32]. Thus, these results suggest that the cells try to bypass the inhibition of the other two ErbB receptors. However, these findings from the transcriptome analysis could not be confirmed by our observations at the protein level on Western blots. Following a 4-day treatment with AffiMabs or controls, we analyzed the protein and phosphorylation levels of the four ErbB receptors and the downstream signaling molecules ERK and AKT. Surprisingly, no distinct differences in phosphorylation or protein expression could be detected (Fig. S12 in the ESM), suggesting that the observed upregulation on transcript level might not translate to the protein level.

Intrigued by the observation of higher transcript levels of the HER3 receptor, we set out to test whether additional blocking of the HER3 receptor could further increase the cytotoxic effect of our AffiMab molecules. To this end, we measured cell viability after a 3-day treatment with increasing concentrations of Tra-zEGFR-L15 AffiMab or Tra-zEGFR-L15 supplemented with equimolar amounts of a HER3-binding Affibody molecule. This Affibody molecule, zHER3, has earlier been described as able to block the interaction of the HER3 receptor with its ligand heregulin and thus inhibit downstream signaling [33]. The addition of soluble zHER3 to our AffiMab did not result in a further reduction of the number of viable cells than what was achieved with the AffiMab alone (Fig. S13 in the ESM). A slight shift in half maximal inhibitory concentration value was observed for the combination treatment of Tra-zEGFRL15 and zHER3 compared with Tra-zEGFR-L15 alone, but this shift was nonsignificant (extra-sum-of-squares $\mathrm{F}$ test, $\mathrm{p}>0.05$ ). This might confirm the earlier hypothesis that HER3 is only overexpressed at the transcript but not the protein level.

\section{Discussion}

In this study, we have described the generation and functional analysis of bispecific AffiMabs. Solid-phase cloning allowed the C-terminal genetic fusion of an EGFR-binding Affibody molecule to either the LC or the HC of the HER2binding therapeutic antibody trastuzumab. The AffiMabs generated in this way recognize their targets, HER2 and EGFR, as both recombinant ECDs and membrane proteins and bind to FcRn (Fig. 1; Fig. S4 in the ESM). Both AffiMabs reduced proliferation of the gastric cancer cell line NCI-N87 more efficiently than trastuzumab or a combination of trastuzumab and zEGFR (Fig. 2; Fig. S5 in the ESM). To understand the mechanism behind this increased cytotoxic effect, we measured the cell surface levels of EGFR and HER2 and analyzed the phosphorylation state and transcript levels and the percentage of apoptotic cells.

Results from transcriptome analysis following the different treatments showed that the AffiMab molecules enhanced the effect of trastuzumab. Genes relating to proliferation, DNA replication, and repair, glycolysis, and cell cycle were downregulated in cells treated with AffiMabs compared with those treated with trastuzumab (Fig. 4; Fig. S10 in the ESM). This could explain the lower viable cell density observed in the AffiMab-treated cells. Interestingly, pathways relating to cancer, particularly genes associated with the ErbB signaling pathway, were upregulated after AffiMab treatment (Fig. 4; Figs. S10 and S3 in the ESM). This might indicate that the cells try to compensate for the reduced signaling through EGFR and HER2 by overexpressing, for example, HER3 and PI3K. This theory was tested in another proliferation analysis, this time with a HER3 inhibitor in addition to the AffiMab molecule (Fig. S13 in the ESM). However, no difference in antiproliferative behavior was detected between the AffiMab and the AffiMab supplemented with a HER3inhibiting Affibody. Given the Affibody we used was earlier described as inhibiting heregulin-induced cancer cell growth [33], supplementation of the growth medium with heregulin might have yielded a clearer result [34]. Another potential explanation is provided by the finding from Western blot analysis (Fig. S12 in the ESM) that no differences in protein expression or phosphorylation of the ErbB receptors and selected downstream signaling molecules were detected, which in turn can potentially be accounted for by the fact that genes essential for protein synthesis were also downregulated upon AffiMab treatment. In conclusion, it can be hypothesized that the cells are trying to compensate for the blocking of EGFR and HER2 through upregulation of HER3, but this remains ineffective because of impaired protein synthesis. Our data, from both the transcriptomics analysis and the propidium iodide and annexin $\mathrm{V}$ staining could not establish a major influence of apoptosis on the observed cytotoxic effect.

Strikingly, although zEGFR did not show any cytotoxic effect on its own or in combination with trastuzumab, the genetic fusion of zEGFR to trastuzumab conferred an additive cytotoxic effect. The clearest difference between a combination treatment and AffiMab treatment is the ability of the AffiMab to downregulate EGFR surface expression. According to flow cytometric measurement, AffiMabs reduced surface HER2 as much as trastuzumab did but also reduced surface EGFR, which the combination of trastuzumab and zEGFR did not achieve (Fig. 3). Unfortunately, we were unable to confirm this observation using confocal microscopy, possibly because of the inherent limitations of the method, because only a limited number of cells were 
analyzed, or because an insufficient magnification was used. For EGFR, the low surface expression in the untreated state rendered an optical detection of reduced expression difficult.

The transcriptomics analysis did not indicate a transcriptional regulation of EGFR expression, but other possible ways to explain the reduced surface levels remain. Lowered EGFR levels can be due to reduced protein synthesis, a theory supported by the transcriptome data, or increased protein degradation, the latter being a reported mechanism of action for trastuzumab on HER2 [35]. Also, a higher endocytosis rate combined with impaired recycling, which Spangler et al. [30] found to be a mode of action of their multispecific EGFR binder, could explain the observed reduction in EGFR levels. We consider it likely that all these processes (reduced protein synthesis, increased degradation, and impaired recycling) contribute to the observed effect of lower EGFR surface levels. With EGFR surface levels on NCI-N87 being low from the start compared with HER2 levels, the increase in endocytosed EGFR or reduction of total EGFR presumably is too small to be detected by confocal microscopy or Western blot, respectively.

\section{Conclusions}

The present study has shown that AffiMab molecules potentiate the effect of trastuzumab in inhibiting cancer cell growth; however, to corroborate the therapeutic advantage of these molecules, the cytotoxic effect should be confirmed on primary cancer cells displaying different levels of EGFR and HER2 to establish how receptor density influences the cytotoxic effect. Moreover, we envision future studies assessing the in vivo effect of our molecules as well as in vivo stability and pharmacokinetics.

Overall, our results highlight the potential of the AffiMab format in therapeutic applications. The solid-phase cloning technique we employed, with its inherent modularity, allows the simple generation of AffiMabs with other therapeutically relevant target specificities, e.g. HER2 and IGF1R, as well as the generation of multivalent or multispecific molecules similar to the proteins generated by LaFleur et al. [18]. With regards to the transcriptional upregulation of HER3 and the findings by Spangler et al. [30] that multispecific antibodies induce a higher degree of cross-linking, resulting in increased receptor internalization, a trispecific molecule targeting EGFR, HER2, and HER3 would be of interest. Previous studies have highlighted the importance of linker length and orientation of the Affibody molecule in a fusion protein $[19,36]$, thus motivating further optimization of the AffiMab format with regards to linker length and Affibody molecule direction, which again is simplified by the modular nature of the solid-phase cloning technique. Also, based on our results from the FcRn binding, which indicate that the $\mathrm{Fc}$ region is accessible, an analysis of the ability of AffiMabs to elicit antibody-dependent cell-mediated cytotoxicity, complement-dependent cytotoxicity, and antibody-dependent phagocytic cytotoxicity will be interesting topics for future investigation as an intact $\mathrm{Fc}$ region is an asset of AffiMabs compared with other bispecific protein formats. The presented results and possibilities for the bispecific HER2-EGFR-AffiMabs also highlight the potential of this bispecific format as a prospective therapeutic and as a means to elucidate cancer biology.

Supplementary Information The online version contains supplementary material available at https://doi.org/10.1007/s40268-021-00339-2.

Acknowledgements The authors thank M. Hjelmare, M. Wiking, and E. Lundberg for their help with the confocal microscopy experiment and J. Seijsing for help with the FcRn-binding analysis.

\section{Declarations}

Funding This work was supported by KTH-Royal Institute of Technology, Vinnova Sweden's Innovation Agency through research centers CellNova, AAVNova and AdBIOPRO, SSF Swedish Foundation for Strategic Research, NNF Center for Biosustainability, WCPR-Wallenberg Center for Protein Research, and the Knut and Alice Wallenberg Foundation

Conflicts of interest BK Ko, HJ Lee, KT Kim and JS Lee are employees at AbClon Inc. FY Frejd is employed at Affibody AB. AL Volk, A Mebrahtu, M Lundqvist, M Karlander, and J Rockberg have no conflicts of interest that are directly relevant to the content of this article.

Ethics approval Not applicable.

Consent to participate Not applicable.

Consent for publication Not applicable.

Availability of data and material Further information and requests for resources and reagents should be directed to and will be fulfilled by the corresponding author. All reagents generated in this study are available from the corresponding author with a completed materials transfer agreement. The transcriptomic dataset supporting the current study has been deposited on Mendeley Data and can be reached through the following https://doi.org/10.17632/k3h2hwn9p5.1.

Code availability All software and code used for transcriptomic analysis as described in the Materials and Methods section is publicly available.

Author contributions The study was designed by ALV, FYF, KTK, JSL, and JR. Data acquisition, analysis, and interpretation were performed by ALV, AM, BKK, ML, MK, HJL, and JR. The manuscript was written by ALV, AM, ML, MK, BKK, and JR. All authors reviewed the manuscript and approved the final version of the manuscript.

Open Access This article is licensed under a Creative Commons Attribution-NonCommercial 4.0 International License, which permits any non-commercial use, sharing, adaptation, distribution and reproduction in any medium or format, as long as you give appropriate credit to the original author(s) and the source, provide a link to the Creative 
Commons licence, and indicate if changes were made. The images or other third party material in this article are included in the article's Creative Commons licence, unless indicated otherwise in a credit line to the material. If material is not included in the article's Creative Commons licence and your intended use is not permitted by statutory regulation or exceeds the permitted use, you will need to obtain permission directly from the copyright holder. To view a copy of this licence, visit http://creativecommons.org/licenses/by-nc/4.0/.

\section{References}

1. Tebbutt N, Pedersen MW, Johns TG. Targeting the ERBB family in cancer: couples therapy. Nat Rev Cancer. 2013;13:663-73.

2. Roskoski R. The ErbB/HER family of protein-tyrosine kinases and cancer. Pharmacol Res. 2014;79:34-74.

3. Bang Y-J, Van Cutsem E, Feyereislova A, Chung HC, Shen L, Sawaki A, et al. Trastuzumab in combination with chemotherapy versus chemotherapy alone for treatment of HER2-positive advanced gastric or gastro-oesophageal junction cancer (ToGA): a phase 3, open-label, randomised controlled trial. Lancet. Elsevier Ltd. 2010;376:687-97.

4. Slamon DJ, Leyland-Jones B, Shak S, Fuchs H, Paton V, Bajamonde A, et al. Use of chemotherapy plus a monoclonal antibody against HER 2 for metastatic breast cancer that overexpresses HER2. N Engl J Med. 2001;344:783-92.

5. Nahta R, Esteva FJ. HER2 therapy: molecular mechanisms of trastuzumab resistance. Breast Cancer Res. 2006;8:215.

6. Pohlmann PR, Mayer IA, Mernaugh R. Resistance to trastuzumab in breast cancer. Clin Cancer Res. 2009;15:7479-91.

7. Vu T, Claret FX. Trastuzumab: updated mechanisms of action and resistance in breast cancer. Front Oncol. 2012;2:62.

8. Narayan M, Wilken JA, Harris LN, Baron AT, Kimbler KD, Maihle NJ. Trastuzumab-induced HER reprogramming in "resistant" breast carcinoma cells. Cancer Res. 2009;69:2191-4.

9. Henjes F, Bender C, von der Heyde S, Braun L, Mannsperger H a, Schmidt C, et al. Strong EGFR signaling in cell line models of ERBB2-amplified breast cancer attenuates response towards ERBB2-targeting drugs. Oncogenesis. Nature Publishing Group; 2012;1:e16.

10. Ritter CA, Perez-Torres M, Rinehart C, Guix M, Dugger T, Engelman JA, et al. Human breast cancer cells selected for resistance to trastuzumab in vivo overexpress epidermal growth factor receptor and ErbB ligands and remain dependent on the ErbB receptor network. Clin Cancer Res. 2007;13:4909-19.

11. Larbouret $\mathrm{C}$, Gaborit N, Chardès T, Coelho M, Campigna E, Bascoul-Mollevi $\mathrm{C}$, et al. In pancreatic carcinoma, dual EGFR/HER2 targeting with cetuximab/trastuzumab is more effective than treatment with trastuzumab/erlotinib or lapatinib alone: implication of receptors' down-regulation and dimers' disruption. Neoplasia. 2012;14:121-30.

12. Kawaguchi Y, Kono K, Mimura K, Mitsui F, Sugai H, Akaike H, et al. Targeting EGFR and HER-2 with cetuximab- and trastuzumab-mediated immunotherapy in oesophageal squamous cell carcinoma. Br J Cancer. 2007;97:494-501.

13. Zheng L, Tan W, Zhang J, Yuan D, Yang J, Liu H. Combining trastuzumab and cetuximab combats trastuzumab-resistant gastric cancer by effective inhibition of EGFR/ErbB2 heterodimerization and signaling. Cancer Immunol Immunother. Springer Berlin Heidelberg; 2014;63:581-6.

14. Ko B-K, Lee S-Y, Lee Y-H, Hwang I-S, Persson H, Rockberg J, et al. Combination of novel HER2-targeting antibody 1E11 with trastuzumab shows synergistic antitumor activity in HER2-positive gastric cancer. Mol Oncol. 2014;
15. Rockberg J, Schwenk JM, Uhlén M. Discovery of epitopes for targeting the human epidermal growth factor receptor 2 (HER2) with antibodies. Mol Oncol. 2009;3:238-47.

16. Trivedi A, Stienen S, Zhu M, Li H, Yuraszeck T, Gibbs J, et al. Clinical pharmacology and translational aspects of bispecific antibodies. Clin Transl Sci. Wiley-Blackwell. 2017;10:147-62.

17. Ståhl S, Gräslund T, Eriksson Karlström A, Frejd FY, Nygren P-Å, Löfblom J. Affibody molecules in biotechnological and medical applications. Trends Biotechnol. 2017;35:691-712.

18. LaFleur DW, Abramyan D, Kanakaraj P, Smith RG, Shah RR, Wang $\mathrm{G}$, et al. Monoclonal antibody therapeutics with up to five specificities: functional enhancement through fusion of targetspecific peptides. MAbs. 2013;5:208-18.

19. Yu F, Gudmundsdotter L, Akal A, Gunneriusson E, Frejd F, Nygren P-A. An affibody-adalimumab hybrid blocks combined IL-6 and TNF-triggered serum amyloid A secretion in vivo. MAbs. Taylor \& Francis. 2014;6:1598-607.

20. Friedman M, Lindström S, Ekerljung L, Andersson-Svahn H, Carlsson J, Brismar H, et al. Engineering and characterization of a bispecific HER2 x EGFR-binding affibody molecule. Biotechnol Appl Biochem. 2009;54:121-31.

21. Ekerljung L, Wållberg H, Sohrabian A, Andersson K, Friedman M, Frejd FY, et al. Generation and evaluation of bispecific affibody molecules for simultaneous targeting of EGFR and HER2. Bioconjug Chem. American Chemical Society. 2012;23:1802-11.

22. Lundqvist M, Edfors F, Sivertsson $\AA$, Hallström BM, Hudson EP, Tegel H, et al. Solid-phase cloning for high-throughput assembly of single and multiple DNA parts. Nucleic Acids Res. Oxford University Press; 2015;43:e49.

23. Bray NL, Pimentel H, Melsted P, Pachter L. Near-optimal probabilistic RNA-seq quantification. Nat Biotechnol. 2016;34:525-7.

24. Love MI, Huber W, Anders S. Moderated estimation of fold change and dispersion for RNA-seq data with DESeq2. Genome Biol. BioMed Central; 2014;15:550.

25. Väremo L, Nielsen J, Nookaew I. Enriching the gene set analysis of genome-wide data by incorporating directionality of gene expression and combining statistical hypotheses and methods. Nucleic Acids Res. Oxford University Press. 2013;41:4378-91.

26. Liberzon A. A Description of the Molecular Signatures Database (MSigDB) Web Site. Methods Mol Biol. 2014. p. 153-60.

27. Luo W, Brouwer C. Pathview: an R/Bioconductor package for pathway-based data integration and visualization. Bioinformatics. Oxford Academic. 2013;29:1830-1.

28. Friedman M, Orlova A, Johansson E, Eriksson TLJ, Höidén-Guthenberg I, Tolmachev V, et al. Directed evolution to low nanomolar affinity of a tumor-targeting epidermal growth factor receptor-binding affibody molecule. J Mol Biol. 2008;376:1388-402.

29. Yang D, Kuan CT, Payne J, Kihara A, Murray A, Wang LM, et al. Recombinant heregulin-Pseudomonas exotoxin fusion proteins: interactions with the heregulin receptors and antitumor activity in vivo. Clin Cancer Res. 1998;4.

30. Spangler JB, Manzari MT, Rosalia EK, Chen TF, Wittrup KD. Triepitopic antibody fusions inhibit cetuximab-resistant BRAF and KRAS mutant tumors via EGFR signal repression. J Mol Biol. Elsevier Ltd. 2012;422:532-44.

31. Sergina NV, Rausch M, Wang D, Blair J, Hann B, Shokat KM, et al. Escape from HER-family tyrosine kinase inhibitor therapy by the kinase-inactive HER3. Nature. NIH Public Access. 2007;445:437-41.

32. Yu X, Ghamande S, Liu H, Xue L, Zhao S, Tan W, et al. Targeting EGFR/HER2/HER3 with a Three-in-One Aptamer-siRNA Chimera Confers Superior Activity against HER2 + Breast Cancer. Mol Ther Nucleic Acids. Cell Press; 2018;10:317-30. 
33. Göstring L, Malm M, Höidén-Guthenberg I, Frejd FY, Ståhl S, Löfblom J, et al. Cellular effects of HER3-specific affibody molecules. PLoS One. Public Library of Science; 2012;7:e40023.

34. Geuijen CAW, De Nardis C, Maussang D, Rovers E, Gallenne T, Hendriks LJA, et al. Unbiased combinatorial screening identifies a bispecific IgG1 that potently inhibits HER3 signaling via HER2guided ligand blockade. Cancer Cell. Elsevier; 2018;33:922-936. e10.
35. Klapper LN, Waterman H, Sela M, Yarden Y. Tumor-inhibitory antibodies to HER-2/ErbB-2 may act by recruiting c-Cbl and enhancing ubiquitination of HER-2. Cancer Res. 2000;60:3384-8.

36. Silacci M, Baenziger-Tobler N, Lembke W, Zha W, Batey S, Bertschinger J, et al. Linker length matters, fynomer-Fc fusion with an optimized linker displaying picomolar IL-17A inhibition potency. J Biol Chem. 2014;289:14392-8. 